Pacific Journal of Mathematics

COMPACTNESS OF MAPPINGS 


\title{
COMPACTNESS OF MAPPINGS
}

\author{
EDWIN DUDA
}

\begin{abstract}
A class of spaces that admit to a special kind of mapping onto the nonnegative real numbers is considered and it is shown that this particular type of space is invariant under compact monotone mappings. It is also shown that if such a space admits to a one to one (monotone) mapping onto a "nice" subset of the plane then the mapping is a homeomorphism (compact monotone mapping). If the one to one mapping (monotone) is not a homeomorphism (compact monotone mapping) then its range necessarily separates $E^{2}$.
\end{abstract}

In [3] the author showed that if a locally connected generalized continuum $X$ with the property that the complement of each compact set has only one nonconditionally compact component admits a one-toone mapping $f$ onto $E^{2}$, then $f$ is a homeomorphism. This result was then used in [4] to show that reflexive compact mappings of $E^{2}$ onto $E^{2}$ are compact mappings. This condition that the domain be all of $E^{2}$ and the range be all of $E^{2}$ are naturally unsatisfactory conditions. In this paper we undertake to reduce these conditions by considering spaces which admit to certain special kinds of mappings onto the nonnegative real numbers. It is shown that these spaces include numerous subsets of $E^{2}$ and also include spaces which are not subsets of $E^{2}$. In general a completely satisfactory reduction on the hypotheses used for the main result in [4] is obtained.

2. Notation and definition. Throughout $X$ and $Y$ will be metric spaces and mapping will mean continuous function. A locally connected generalized continuum is a connected separable metric space which is locally compact and locally connected. A mapping is monotone $f$ each point inverse is a continuum and a mapping is compact if the inverse image of each compact set is also compact. A continuum $X$ is unicoherent if whenever $X=A \cup B$, where $A$ and $B$ are continua, then so is $A \cap B$. The nonnegative real numbers will be represented by $[0, \infty)$ and $[a, b]$ will be the real numbers interval from $a$ to $b$ or a simple arc joining $a$ to $b$ depending on the context. The cyclic element theory used is that of reference [10]. A topological ray is a homeomorphic image of a ray in the real line. A set $B$ in a metric space is conditionally compact if its closure is a compact set. The Euclidean plane will be represented by $E^{2}$.

3. Preliminary results. 
THEOREM 1. Let $X$ be a noncompact metric space which has the representation $X=\bigcup_{i=1}^{\infty} A_{i}$, where $A_{i} \subset \operatorname{int} A_{i+1}, i=1,2,3, \cdots$ and each $A_{i}$ is a locally connected unicoherent continuum which does not separate $X$. Then there is a mapping $g$ of $X$ onto $[0, \infty)$ and $a$ monotone increasing sequence of positive real numbers $a_{i},\left(a_{i}\right) \rightarrow \infty$, such that $g^{-1}\left[0, a_{i}\right]$ is a locally connected unicoherent continuum for $i=1,2,3, \cdots$.

Proof. For convenience of notation assume int $A_{1} \neq \phi$. Let $B_{i}=$ $A_{i} \cap C l\left(X-A_{i}\right)$ for each positive integer $i$. Each $B_{i}$ is a continuum, [5], the $B_{i}$ are pairwise disjoint and the union of any subcollection of $B_{i}$ is a closed subset. By a modified version of Urysohn's Lemma there is a mapping $f_{1}$ of $A_{1}$ onto $[0,1]$ with $f_{1}\left(B_{1}\right)=1, f_{1}^{-1} f_{1}\left(B_{1}\right)=B_{1}$. Similarly there is a mapping $f_{2}$ of $B_{1} \cup A_{2}-A_{1}$ onto $[1,2]$ with

$$
f_{2}\left(B_{1}\right)=1, f_{2}^{-1} f_{2}\left(B_{1}\right)=B_{1}, f_{2}\left(B_{2}\right)=2, f_{2}^{-1} f_{2}\left(B_{2}\right)=B_{2} \text {. }
$$

In general there exists a mapping $f_{n}$ of $B_{n-1} \cup A_{n}-A_{n-1}$ onto $[n-1, n]$ with $f_{n}\left(B_{n-1}\right)=n-1, f_{n}^{-1} f_{n}\left(B_{n-1}\right)=B_{n-1}, f_{n}\left(B_{n}\right)=n, f_{n}^{-1} f_{n}\left(B_{n}\right)=B_{n}$. Let $g$ be the mapping of $X$ onto $[0, \infty)$ which is defined in the following manner. For $x \in A_{j}$, set $g(x)=f_{j}(x)$ and note that $g$ is a mapping with the required properties.

There is a partial converse to theorem one which is useful in identifying some of the properties of spaces which admit to a special kind of mapping onto $[0, \infty)$.

THEOREM 2. Let $X$ be a metric space which admits a mapping $g$ onto $[0, \infty)$ for which there exists a strictly monotone increasing sequence of positive real numbers $a_{i},\left(a_{i}\right) \rightarrow \infty$, such that $g^{-1}\left[0, a_{i}\right]$ is a locally connected unicoherent continuum and $g^{-1}\left[a_{i}\right]$ is a continuum for each positive integer $i$. Then $X$ has the representation $X=$ $\bigcup_{i=1}^{\infty} A_{i}$, where $A_{i} \subset \operatorname{int} A_{i+1}$, each $A_{i}$ in a locally connected unicoherent continuum, and $g^{-1}\left[a_{i}, \infty\right)$ is connected for $i=1,2,3, \cdots$.

Proof. Let $A_{i}=g^{-1}\left[0, a_{i}\right]$ for each positive integer $i$. Clearly $A_{i} \subset \operatorname{int} A_{i+1}$ for each positive integer $i$ and since $g^{-1}\left[a_{i}\right]$ is a continuum it follows that $g^{-1}\left[a_{i}, \infty\right)$ is connected.

Another usuful version of theorems one and two is possible for monotone mappings. First we establish a useful lemma of which there are more general versions, i.e., Theorem 2 of [9].

Lemma. Let $X$ be a locally connected generalized continuum. If $g$ is a monotone mapping of $X$ onto $[0, \infty)$ then $f$ is a compact 
mapping.

Proof. Let $K$ be a compact subset of $[0, \infty)$ with least upper bound $u$ and greatest lower bound $l$. There is a continuum $K_{0}$ containing $g^{-1}(u) \cup g^{-1}(l)$. The closed interval $g\left(K_{0}\right)$ contains $K$ so that $g^{-1} g\left(K_{0}\right)$ contains $g^{-1}(K)$. By monotoneness of $g, g^{-1} g\left(K_{0}\right)$ is a continuum so that $g^{-1}(K)$ is compact.

THEOREM 3. Let $X$ be a locally connected generalized continuum and $g$ a monotone mapping of $X$ onto $[0, \infty)$. If there exists $a$ sequence of positive real numbers $a_{i},\left(a_{i}\right) \rightarrow \infty$, such that each $g^{-1}\left[0, a_{i}\right]$ is embeddable in $E^{2}$ and no point inverse of $g$ separates $E^{2}$ in some embedding, then $g^{-1}\left[0, a_{i}\right]$ fails to separate $E^{2}$ for each positive integer $i$.

Proof. It is well known that if one embedding of a continuum in $E^{2}$ fails to separate $E^{2}$ then the same is true for all other embeddings. Suppose then that $g^{-1}\left[0, a_{i}\right]$ is a subset of $E^{2}$ under some embedding. Let $G$ be the decomposition of $E^{2}$ into the points not in $g^{-1}\left[0, a_{i}\right]$ and the continua $g^{-1}[c], 0 \leq c \leq a_{i}$. Since this decomposition is upper semicontinuous it follows by a well-known theorem of R. L. Moore, [6], that the decomposition space $Y$ is a topological plane. Furthermore the image of $g^{-1}\left[0, a_{i}\right]$ under the natural mapping $\psi$ of the decomposition $G$ is a simple arc. Since the arc $\psi\left(g^{-1}\left[0, a_{i}\right]\right)$ does not separate $Y$ and $\psi$ is a monotone compact mapping, $g^{-1}\left[0, a_{i}\right]$ does not separate $E^{2}$.

It will be useful for further discussion and statements of results to refer to a locally connected generalized continuum which admits to a mapping $g$ of the type in Theorems 1, 2, or 3 as a $g$-unicoherent space. To give some indication of a class of spaces which satisfy the conditions of Theorem 1 recall that a locally connected continuum each true cyclic element of which is homeomorphic to a 2-sphere is called a cactoid. It is well known that a monotone image of a 2-sphere is a cactoid and that any cactoid can be realized by a monotone mapping defined on a 2-sphere. Moreover, any monotone image of a cactoid is also a cactoid. The space obtained by removing a noncut point from a cactoid satisfies the conditions of Theorem 1. (See Theorem 4.)

A base set is a locally connected continuum which is homeomorphic to a continuum in $E^{2}$ every true cyclic element of which is a 2-cell. A hemi-cactoid is a locally connected continuum which is the union of a base set and disjoint sequence of cactoids each intersecting the base set in a single point and subject to the condition that for any $\varepsilon>0$ at most a finite number of the cactoids have diameter greater than $\varepsilon$. It is well known that any monotone image of a hemi-cactoid is also a hemi-cactoid and any hemi-cactoid can be realized by a monotone mapping defined on a 2-cell. The space obtained by removing a noncut 
point from a hemi-cactoid will satisfy the conditions of Theorem 1 provided the noncut point is not in an open 2-cell of a true cyclic element which is a 2-cell. (See Theorem 4).

We generalize the notion of cactoid and hemi-cactoid by considering a locally connected continuum $X$ in which each true cyclic element is either a 2-sphere or a closed 2-cell. Before establishing the main theorem concerning such spaces consider the following remarks. For a 2-sphere $S$ and any point $p \in S, S-p=\bigcup_{i=1}^{\infty} A_{i}$, where $A_{i}$ is a closed 2-cell, $A_{i} \subset \operatorname{int} A_{i+1}$, and $(S-p)-A_{i}$ is connected for each positive integer. For a closed 2-cell $C$ and any point $p$ on the boundary of $C, C-p=\bigcup_{i=1}^{\infty} A_{i}$, where $A_{i}$ is a closed 2-cell, $A_{i} \subset \operatorname{int} A_{i+1}$, and $(C-p)-A_{i}$ is connected for each positive integer $i$.

Much of the preceding discussion of known results can be found in [10].

THEOREM 4. Let $X$ be a locally connected continuum every true cyclic element of which is a closed 2-cell or a 2-sphere. If $p$ is a noncut point, $p$ not in an open 2-cell of a true cyclic element which is a closed 2-cell, then $X-p=\bigcup_{i=1}^{\infty} A_{i}$, where $A_{i}$ is a unicoherent locally connected continuum, $A_{i} \subset \operatorname{int} A_{i+1}$, and $X-A_{i}$ is connected for each positive integer $i$.

Proof. Suppose $p$ is in a true cyclic element $S$ which is a 2-sphere. Let $S-p=\bigcup_{i=1}^{\infty} A_{1}^{\prime}$, where $A_{1}^{\prime}$ is a closed 2-cell, $A_{1}^{\prime} \subset \operatorname{int} A_{1+1}^{\prime}$, and $(S-p)-A_{1}^{\prime}$ is connected for each positive integer $i$. For each positive integer $i$, let $A_{i}$ be $A_{i}^{\prime}$ union all of the components of $X-S$ whose closure meets $A_{i}^{\prime}$. This gives the required representation for $X-p$.

In the case where $p$ is in a closed 2-cell which is a true cyclic element, the argument is similar.

Suppose now that $p$ is a noncut point which does not belong to a true cyclic element. For $q \in X, q \neq p$, there is a simple arc $[q, p]$ joining $q$ to $p$. Let $K$ be true cyclic element which has a nondegenerate intersection with $[q, p]$. The set $K \cap[q, p]$ is a simple arc and hence has a last point in the order $q, p$. Let $H$ be the set of such points obtained by considering all nondegenerate intersections of true cyclic elements with $[q, p]$ and let $b$ be the least upper bound of $H$ on $[q, p]$, (take $b=q$ if $H=\phi)$. If $b=p$, then $H$ contains a sequence $\left(p_{i}\right)$ converging to $p$ and if $b \neq p$, then choose $\left(p_{i}\right)$ to be any sequence on $[b, q]$ converging to $p$. It can be assumed that the sequence $\left(p_{i}\right)$ is monotonic increasing in the order $q, p$. For each positive integer $i$ define $A_{i}$ to be $p_{i}$ union of all the components of $X-p_{i}$ except the one that contains $\left(p_{i}, p\right]$. For each positive integer $A_{i}$ is a unicoherent locally connected continuum, $A_{i} \subset \operatorname{int} A_{i+1}$, and $X-A_{i}$ is connected. 
In order to make the statements of some theorems somewhat briefer, it is convenient to make the following definition. A space $X$ is said to be a $D$-cactoid if $X$ is a locally connected generalized continuum which is obtained by removing a noncut point $p, p$ not in an open two cell of a true cyclic element which is a 2-cell, from a locally connected continuum each true cyclic element of which is a 2-sphere or a 2-cell.

CoRollary 1. Every D-cactoid is a g-unicoherent space.

THEOREM 5. If $f(X)=Y$ is a compact monotone mapping and $X$ is a D-cactoid then so is $Y$.

Proof. Let $X^{\prime}$ be the locally connected continuum and $p$ the noncut point for which $X^{\prime}-p=X$. The space $Y$, since $f$ is a compact mapping, is known to be a locally connected generalized continuum. Denote by $Y^{\prime}$ the one point compactification of $Y$ and denote the compactifying point by $q, q \notin Y$. There is a natural extension of $f$, call it $f^{\prime}$, which takes $p$ onto $q$. Let $K$ be a true cyclic element of $Y^{\prime}$ and note by the monotoneness of $f^{\prime}, f^{\prime-1}(K)$ is a continuum. There is a true cyclic element $C$ of $X^{\prime}$ with $f^{\prime}(C) \supset K$. The mapping $f^{\prime} / C$ is monotone hence $f^{\prime}(C)$ is a cactoid or a hemi-cactoid. Clearly $K$ is a true cyclic element of $f^{\prime}(C)$ and as such is a 2-sphere or a 2-cell. Thus the space $Y^{\prime}-q=Y$ is a $D$-cactoid.

4. Main theorem. In order to make use of a theorem in [11] and portions of the proof of the theorem in [3] the following notation is useful. Let $f(X)=Y$ be a one to one mapping of one locally compact separable metric space $X$ onto another such space $Y$. Let $S$ be the set of points in $X$ at which $f$ is a local homeomorphism and let $T$ be its complement. From a result in [9] the set $S$ is open, $T$ is closed, and $f(S)$ is an open dense set in $Y$. The sets $S$ and $T$ and the following theorem of [11] will be used in proving the main theorems.

Theorem A. Let $X$ be a locally compact arcwise connected separable metric space, let $Y$ be a locally connected generalized continuum. If $f(X)=Y$ is a one to one continuous function which is not a homeomorphism, then there exists a topological ray $R$ in $X$, $R$ closed in $X$, with $f(R)$ a simple closed curve in $Y$. Moreover, if $r$ is the initial point of $R$, there is a subray $R^{\prime}$ of $R$ such that $f\left(R^{\prime} \cup r\right)$ is a simple arc and $R^{\prime} \subset S$.

Theorem 6. Let $X$ be a g-unicoherent space. If $f(X)=Y$ is a 
one-to-one mapping, where $Y \subset E^{2}$ is locally compact, locally connected, and $Y=\bigcup_{i=1}^{\infty} B_{i}, B_{i} \subset \operatorname{int} B_{i+1}(\operatorname{rel} Y), i=1,2,3, \cdots$ and no $B_{i}$ separates $E^{2}$, then $f$ is a homeomorphism.

Proof. The proof is made by proving a series of statements which determine the cyclic structure of the space $X$ if $f$ is supposed not to be a homeomorphism and then reaching a contradiction. Let $a_{i}$ be a sequence of positive real numbers for which $g^{-1}\left[0, a_{i}\right]$ is a locally connected unicoherent continuum and hence $f g^{-1}\left[0, a_{i}\right]$ does not separate $E^{2}$ for any $i$ or as in Theorem 3 each $f^{-1}[c], 0 \leq c<\infty$, fails to separate $E^{2}$ so that each $f g^{-1}\left[0, a_{i}\right]$ fails to separate $E^{2}$ for any sequence $a_{i},\left(a_{i}\right) \rightarrow \infty$.

We first show that every simple closed curve $J$ in $X$ bounds an open two cell which is an open subset of $X$. To this end, let $a_{n}$ be an element of the sequence $a_{i}$ for which $g(J) \subset\left[0, a_{n}\right]$. The continuum $f g^{-1}\left[0, a_{n}\right]$ fails to separate $E^{2}$ and contains the simple closed curve $f(J)$ so it contains the open two cell bounded by $f(J)$. Since $f / g^{-1}\left[0, a_{n}\right]$ is a homeomorphism onto its image, $f^{-1}(E)$ is an open two cell which is open in $X$ and is bounded by $J$.

To show the space $X$ has exactly one nonconditionally compact cyclic element suppose first that all cyclic elements of $X$ are compact. By Theorem A there exists a ray $R, R$ closed in $X$, such that $f(R)$ is a simple closed curve and a subray $R^{\prime} \subset R$ and $R^{\prime} \subset S$. That is, $R^{\prime} \subset S$ implies $f$ is a local homeomorphism at each point of $R^{\prime}$. The closed ray $R^{\prime}$ cannot be a subset of any compact cyclic element so that $R^{\prime}$ contains a point $p$ which separates $X$. Let $X_{0}$ be $R$ union all the components of $X-R$ that map into the open two cell bounded by $f(R)$. There is an integer $n$ such that $B_{n} \supset f(R)$ consequently $f\left(X_{0}\right)$ is a closed two cell. A contradiction is obtained by nothing that $p$ locally separates $X_{0}$ but does not locally separate $f\left(X_{0}\right)$. Thus $X$ has at least one nonconditionally compact cyclic element. We show that $X$ has exactly one nonconditionally compact cyclic element by showing that the complement of any compact set $K$ has at most one nonconditionally compact component. For a compact set $K$ there is an integer $n$ for which $g(K) \subset\left[0, a_{n}\right)$. The set $g^{-1}\left[0, a_{n}\right]$ is a continuum containing and $g^{-1}\left[a_{n}, \infty\right)$ is connected in all of the cases being considered so that $X-K$ has at most one nonconditionally compact component.

That each nondegenerate compact cyclic element of $X$ is topologically a closed 2-cell and $B=M \cap T$, where $M$ is the noncompact cyclic element of $X$, is a topological line follow from the proofs of (iii) and (v) in [3]. Finally the proof can be completed by using the argument (vi) of [3].

Corollary 1. Let $X$ be a g-unicoherent space and $f(X)=Y a$ 
one-to-one mapping, where $Y \subset E^{2}$ is locally compact and locally connected. If $f$ is not a homeomorphism then $Y$ separates $E^{2}$.

Corollary 2. Let $X$ be a D-cactoid and $f(X)=Y$ a one-to-one mapping, where $Y \subset E^{2}$. If $Y$ is a D-cactoid, then $f$ is a homeomorphism.

5. Applications. In [9] it was proved that any monotone mapping of a plane onto a plane is a compact mapping and in [4] this result was generalized by proving that any reflexive compact mapping of a plane onto a plane is a compact mapping. These results are useful and interesting but suffer from the unnatural condition that the mapping must be an onto mapping. It is the purpose of this section to show that by virtue of the theorems in section four much stronger results can be established. These results generalize corresponding theorems of $K$. Borsuk [2] and K. A. Sitnikov [7].

THEOREM 8. If $X$ is a D-cactoid and $f: X \rightarrow E^{2}$ is monotone, where $f(X)$ is locally connected, locally compact, and $f(X)=\bigcup_{i=1}^{\infty} B_{i}$, $B_{i} \subset$ int $B_{i+1}$, and $E^{2}-B_{i}$ is connected for $i=1,2,3, \cdots$, then $f$ is a compact mapping.

Proof. Let $M$ be the decomposition space generated by $f, \pi$ the natural mapping of $X$ onto $M$ and $h$ the unique one-to-one mapping of $M$ into $E^{2}$. The mapping $\pi$ is compact and monotone so that $M$ is a $D$-coctoid. By Theorem 6 the mapping $h$ is a homeomorphism. Since $f$ is the composition of two compact mappings, it is also compact. See [1], [8].

Corollary 1. Let $X$ be a D-cactoid. If $f(X)=Y$ is a monotone mapping, where $Y \subset E^{2}$ is locally compact, locally connected and $Y$ does not separate $E^{2}$, then $f$ is a compact mapping and $Y$ is a D-coatoid.

Corollary 2. Let $X$ be a D-cactoid. If $f(X)=Y$ is a monotone mapping which is not a compact mapping, where $Y \subset E^{2}$ is locally compact and locally connected, then $Y$ separates $E^{2}$.

Corollary 3. Let $f: E^{2} \rightarrow E^{2}$ be a monotone mapping, where $f\left(E^{2}\right)$ is locally compact and locally connected. If $f\left(E^{2}\right)$ satisfies the conditions of Theorem 8 or $E^{2}-f\left(E^{2}\right)$ is connected or $f\left(E^{2}\right)=E^{2}$ then $f$ is a compact mapping. 


\section{REFERENCES}

1. H. Bauer, Verallemeinerung eines Faktorisierungsatzes vom G. T. Whyburn, Arch. Math. 10 (1959), 373-378.

2. K. Borsuk, Über Stetige Abbildungen der Euklidischen Raume, Fund. Math. 21 (1933), 236-243.

3. E. Duda, A theorem on 1-1 mappings, Pacific J. Math. 19 (1966), 253-257.

4. __ Reflexive compact mappings, Proc. Amer. Math. Soc. 17 (1966), 688-693.

5. S. Eilenberg, Transformations continues en circonference et la topologie du plan, Fund. Math. 26 (1936), 61-112.

6. R. L. Moore, Concerning upper semi-continuous collections of continua, Trans. Amer. Math. Soc. 27 (1925), 416-428.

7. K. A. Sitnikov, On continuous mappings of open sets of Euclidean spaces, Mat. Sb. (63) 31 (1952), 439-458.

8. A. H. Stone, Metrizability of decomposition spaces, Proc Amer. Math. Soc. 7 (1956), 690-700.

9. G. T. Whyburn, Compactness of mappings, Amer. J. Math. 81 (1956), 306-313.

10. - Analytic topology, Amer. Math. Soc. Colloquium Publications, 28 (1942).

11. On compactness of mappings, Proc. Nat. Acad. Sci. U.S.A. 52 (1964), $1426-1431$.

Received August 6, 1968. This research was supported by a grant from the National Science Foundation.

UNIVERSITY OF MIAMI

Coral Gables, Florida 


\section{PACIFIC JOURNAL OF MATHEMATICS}

\section{EDITORS}

H. ROYDEN
Stanford University
Stanford, California

\section{R. R. PHELPS}

University of Washington

Seattle, Washington 98105
J. DUGUNDJI

Department of Mathematics

University of Southern California

Los Angeles, California 90007

\section{RICHARD ARENS}

University of California

Los Angeles, California 90024

\section{ASSOCIATE EDITORS}

E. F. BeCKenbaCh
B. H. NEUMANN

F. WOLF
K. YoshidA

\section{SUPPORTING INSTITUTIONS}

\author{
UNIVERSITY OF BRITISH COLUMBIA \\ CALIFORNIA INSTITUTE OF TECHNOLOGY \\ UNIVERSITY OF CALIFORNIA \\ MONTANA STATE UNIVERSITY \\ UNIVERSITY OF NEVADA \\ NEW MEXICO STATE UNIVERSITY \\ OREGON STATE UNIVERSITY \\ UNIVERSITY OF OREGON \\ OSAKA UNIVERSITY \\ UNIVERSITY OF SOUTHERN CALIFORNIA
}

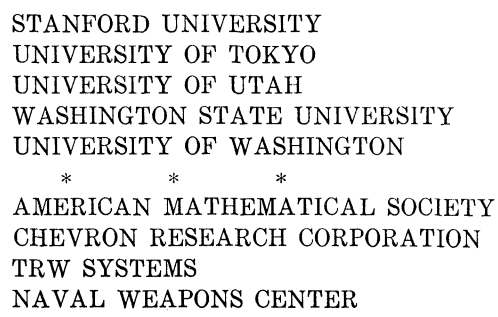

The Supporting Institutions listed above contribute to the cost of publication of this Journal, but they are not owners or publishers and have no responsibility for its content or policies.

Mathematical papers intended for publication in the Pacific Journal of Mathematics should be in typed form or offset-reproduced, double spaced with large margins. Underline Greek letters in red, German in green, and script in blue. The first paragraph or two must be capable of being used separately as a synopsis of the entire paper. It should not contain references to the bibliography. Manuscripts, in duplicate if possible, may be sent to any one of the four editors. Please classify according to the scheme of Math. Rev. 36, 1539-1546. All other communications to the editors should be addressed to the managing editor, Richard Arens, University of California, Los Angeles, California, 90024.

50 reprints are provided free for each article; additional copies may be obtained at cost in multiples of 50 .

The Pacific Journal of Mathematics is published monthly. Effective with Volume 16 the price per volume (3 numbers) is $\$ 8.00$; single issues, $\$ 3.00$. Special price for current issues to individual faculty members of supporting institutions and to individual members of the American Mathematical Society: $\$ 4.00$ per volume; single issues $\$ 1.50$. Back numbers are available.

Subscriptions, orders for back numbers, and changes of address should be sent to Pacific Journal of Mathematics, 103 Highland Boulevard, Berkeley, California, 94708.

PUBLISHED BY PACIFIC JOURNAL OF MATHEMATICS, A NON-PROFIT CORPORATION

Printed at Kokusai Bunken Insatsusha (International Academic Printing Co., Ltd.), 7-17, Fujimi 2-chome, Chiyoda-ku, Tokyo, Japan. 


\section{Pacific Journal of Mathematics \\ Vol. 29, No. $2 \quad$ June, 1969}

Bruce Langworthy Chalmers, On boundary behavior of the Bergman kernel function and related domain functionals ................... 243

William Eugene Coppage, Peirce decomposition in simple Lie-admissible power-associative rings .............................. 251

Edwin Duda, Compactness of mappings...................... 259

Earl F. Ecklund Jr., On prime divisors of the binomial coefficient......... 267

Don E. Edmondson, A modular topological lattice ............... 271

Phillip Alan Griffith, A note on a theorem of Hill ................... 279

Marcel Herzog, On finite groups with independent cyclic Sylow

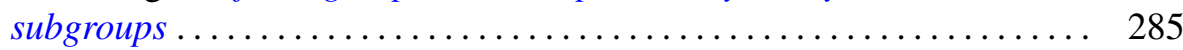

James A. Huckaba, Extensions of pseudo-valuations................. 295

S. A. Huq, Semivarieties and subfunctors of the identity functor ........ 303

I. Martin (Irving) Isaacs and Donald Steven Passman, Finite groups with small character degrees and large prime divisors. II ............ 311

Carl Kallina, A Green's function approach to perturbations of periodic

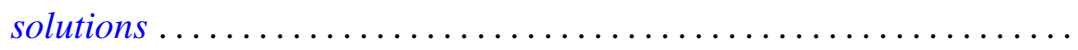

$\mathrm{Al}$ (Allen Frederick) Kelley, Jr., Analytic two-dimensional subcenter manifolds for systems with an integral ....................

Alistair H. Lachlan, Initial segments of one-one degrees ............ 351

Marion-Josephine Lim, Rank k Grassmann products ............. 367

Raymond J. McGivney and William Henry Ruckle, Multiplier algebras of

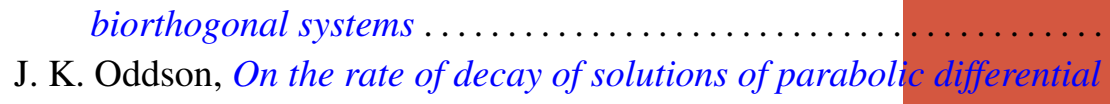

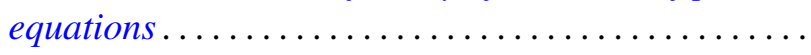

Helmut R. Salzmann, Geometries on surfaces ........... .

Annemarie Schlette, Artinian, almost abelian groups and their groups of automorphisms ............................

Edgar Lee Stout, Additional results on modules over polydisc algebras ...

Lajos Tamássy, A characteristic property of the sphere . .

Mark Lawrence Teply, Some aspects of Goldie's torsion theory. ...

Freddie Eugene Tidmore, Extremal structure of star-shaped sets ...

461

Leon Jarome Weill, Unconditional and shrinking bases in locally convex spaces... 